\title{
Serum apelin levels in polycystic ovary syndrome and its relationship with adiposity profile in females.
}

\footnotetext{
1. MBBS, M.Phil. Lecturer Physiology Jinnah Medical and Dental College.

2. 3rd Year MBBS Student

Medical College, Aga Khan University.

3. MBBS, M.Phil Physiology Karachi Medical and Dental College and University of Karachi.

4. MBBS, M.Phil, Ph.D, FHEA (UK) Biological and Biomedical Sciences Aga Khan University.

Correspondence Address: Dr. Syeda Sadia Fatima Department of Biological and Biomedical Sciences Aga Khan University, Stadium Road, Karachi.

sadia.fatima@aku.edu
}

Article received on: 27/07/2020

Accepted for publication: 26/10/2020

\begin{abstract}
Sassi Kanwal ${ }^{1}$, Ashba Allahwasaya ${ }^{2}$, Nargis Anjum ${ }^{3}$, Syeda Sadia Fatima ${ }^{4}$
ABSTRACT... Objectives: The purpose of the current study was to compare the serum Apelin level in patients with and without polycystic ovarian syndrome to assess this as a diagnostic marker for PCOS. In a case-control study 60 polycystic ovarian syndrome patients diagnosed according to Rotterdam criteria and 20 healthy controls of reproductive age group were recruited. Study Design: Case Control study. Setting: Jinnah Postgraduate Medical Center in Collaboration with Aga Khan University, Karachi. Period: August 2017 and February 2019. Material \& Methods: Blood samples were collected after 12 hours fasting and was used to test serum Apelin, insulin, cortisol and fasting blood glucose levels. Results: Seventy percent PCO subjects had irregular menstrual cycle and $68 \%$ suffered from hirsutism; while all PCO subjects had more than 10 antral follicle seen on ultrasound in either one or both ovaries in comparison of $15 \%$ controls $(p<0.05)$. Higher Apelin and Testosterone levels in PCO group was reported when compared with controls $(p<0.05)$ across all BMI categories. Apelin showed a positive correlation with number of ovarian cysts $(r=0.429 ; p=0.000)$; irregular menstrual cyle $(r=0.276 ; p=0.13)$; blood glucose level $(r=0.270 ; p=0.015)$ and BMI $(r=0.229 ; p=0.14)$. Apelin remained independently associated with the risk of PCOS $(p=0.020)$ and elevated testosterone levels $(p=0.030)$. Conclusion: Serum Apelin showed a strong association with irregular menstruation, hirsutism, ovarian cysts, testosterone and deranged blood glucose levels. Therefore, Apelin appears as a potential source for several risk factor in PCOS women of all BMI types.
\end{abstract}

Key words: $\quad$ Apelin, Hirsutism, Irregular Menstrual, Obesity, PCOS.

Article Citation: Kanwal S, Allahwasaya A, Anjum N, Fatima SS. Serum apelin levels in polycystic ovary syndrome and its relationship with adiposity profile in females. Professional Med J 2021; 28(6):902-906.

https://doi.org/10.29309/TPMJ/2021.28.06.5821

\section{INTRODUCTION}

Polycystic ovary syndrome is the most frequent endocrine ailment in reproductive age group, its frequency is about $6-8 \%$ in the reproductive period. ${ }^{1}$ Worldwide the overall commonness and phenotypic highlights of polycystic ovary disorder (PCOS) is the most widely acknowledged endocrine issue among women of reproductive age. $^{2}$ This difference in prevalence of PCOS around the globe is mainly due to different diagnostic criteria's and because of cultural antithetic groups. Therefore, to overcome this major diagnostic variation differences there is a need to go for ethnic specific guidelines for PCOS. ${ }^{3}$ PCOS is a set of symptoms represented and described by irregular menstrual cycle, sterility, androgen excess and insulin resistance. ${ }^{4}$
PCOS has long lasting hazards such as cardiovascular illnesses, Type-2 DM, dyslipidemia and endometrial carcinoma. ${ }^{5}$

Apelin a newly discovered adipo-cytokine is a cell signaling protein secreted by fatty tissue. apelin (APLN) is a peptide, which is programmed by the APLN gene (endogenic ligand for the G-proteinlinked APJ receptor). ${ }^{6}$ Studies have shown higher apelin levels in PCOS patients with a strong correlation with BMI and insulin resistance. ${ }^{7}$ Therefore, we hypothesize that apelin levels might be increased in PCOS subjects irrespective of their BMI status. This study aims to evaluate and compare serum apelin levels in females with PCOS and controls in order to assess Apelin as a diagnostic marker for PCOS. 


\section{MATERIAL \& METHODS}

This case control study was conducted at BMSI-Jinnah postgraduate medical center in collaboration with Aga Khan University, Karachi between August 2017 and February 2019. Ethical approval was obtained from the institutional review committee (NO.F.2-81-IRB/2017GENL/418/JPMC). A sample size of 80 women was calculated to achieve a power of 90 and an alpha of $5 \%$, with a prevalence of PCOS as $15 \%$ in local population. ${ }^{8}$ Eighty-five subjects agreed to consent and participate in this study. The inclusion criteria was females of reproductive age group diagnosed as PCOS per Rotterdam criteria. Rotterdam criteria for PCOS diagnosis states that a women may present with any 2 out of 3 conditions: Anovulation; hirsutism or less commonly male pattern alopecia or raised free testosterone; or polycystic ovaries on ultrasound (when 10 small antral follicles are seen in each ovary). ${ }^{4}$ Subjects were classified as normal controls if they had no symptom of PCOS as described above. Study participants were selected via convenient sample technique and divided into two groups, Group A: PCO group included individuals with diagnosed polycystic phenotypes $n=60$ and Group B: Control group included individuals without any PCOS phenotypes $n=20$.

Subjects with any systemic diseases like atherosclerosis, diabetes mellitus, hypertension and any other reproductive disorders such as congenital adrenal hyperplasia, androgen secreting tumors, cushing syndrome, thyroid dysfunction and hyper prolactinaemia were excluded from this study. After obtaining written and informed consent from the subjects, their biophysical parameters were measured. Ten milliliters of blood was collected after overnight fasting of 10-12 hours and serum was stored at $-80^{\circ}$ Celsius. Serum samples were used to measure Insulin (cat IN374S by Calbiotech), Cortisol (cat DCMOO1-12) and human Apelin (cat 90291) by enzyme linked immune sorbent assay.

SPSS version 21.0 was used for data entry and exploration. A descriptive statistical analysis of continuous variables was performed. Data on continuous variables i.e. biophysical and biochemical parameters were presented as Mean \pm standard deviation (SD) or \pm standard error of mean (SEM). Student t-test for continuous/ quantitative variables, chi square or Fischer exact test for categorical variables and Pearson coefficient of correlation ( $r$ ) was applied. Multiple regression analysis was performed to identify the effect of Apelin on PCOS. In all statistical analysis only $p$-value $<0.05$ was considered significant.

\section{RESULT}

The groups were age and weight matched therefore no significant difference was observed $(p>0.05)$. However, fasting blood glucose levels in PCOS group was significantly raised as compared to the control group (Table-I). Seventy percent PCOS subjects had irregular menstrual cycle and $68 \%$ suffered from hirsutism; while all PCOS subjects had more than 10 antral follicle seen on ultrasound in either one or both ovaries in comparison of $15 \%$ controls $(p<0.05)$ (Table-l). Higher Apelin and testosterone levels in PCOS group was reported when compared with controls $(p<0.05)$, whereas all other markers showed no difference amongst the groups (Table-II). When we stratified the subjects according the BMI category, higher Apelin levels were seen in subjects with PCOS across all BMI categories. Furthermore, Apelin levels showed a positive correlation with number of ovarian cysts $(r=0.429 ; p=0.000)$; irregular menstrual cyle $(r=0.276 ; p=0.13)$; blood glucose level $(r=0.270$; $p=0.015)$ and $B M I(r=0.229 ; p=0.14)$. When we applied multiple regressions to adjust for the effect of BMI on Apelin levels, Apelin remained independently associated with the risk of PCOS $(p=0.020)$.

\section{DISCUSSION}

Polycystic ovary syndrome (PCOS) is considered as a multifaceted syndrome that causes major health problems such as menstrual irregularities, infertility, excess hair, acne, obesity etc. ${ }^{9}$ The true mechanism of the syndrome still is not fully understood, but literature hints for a genetic link. ${ }^{10}$ In this current study significantly higher level of serum apelin was found in PCOS than normal controls $(p<0.05)$. 


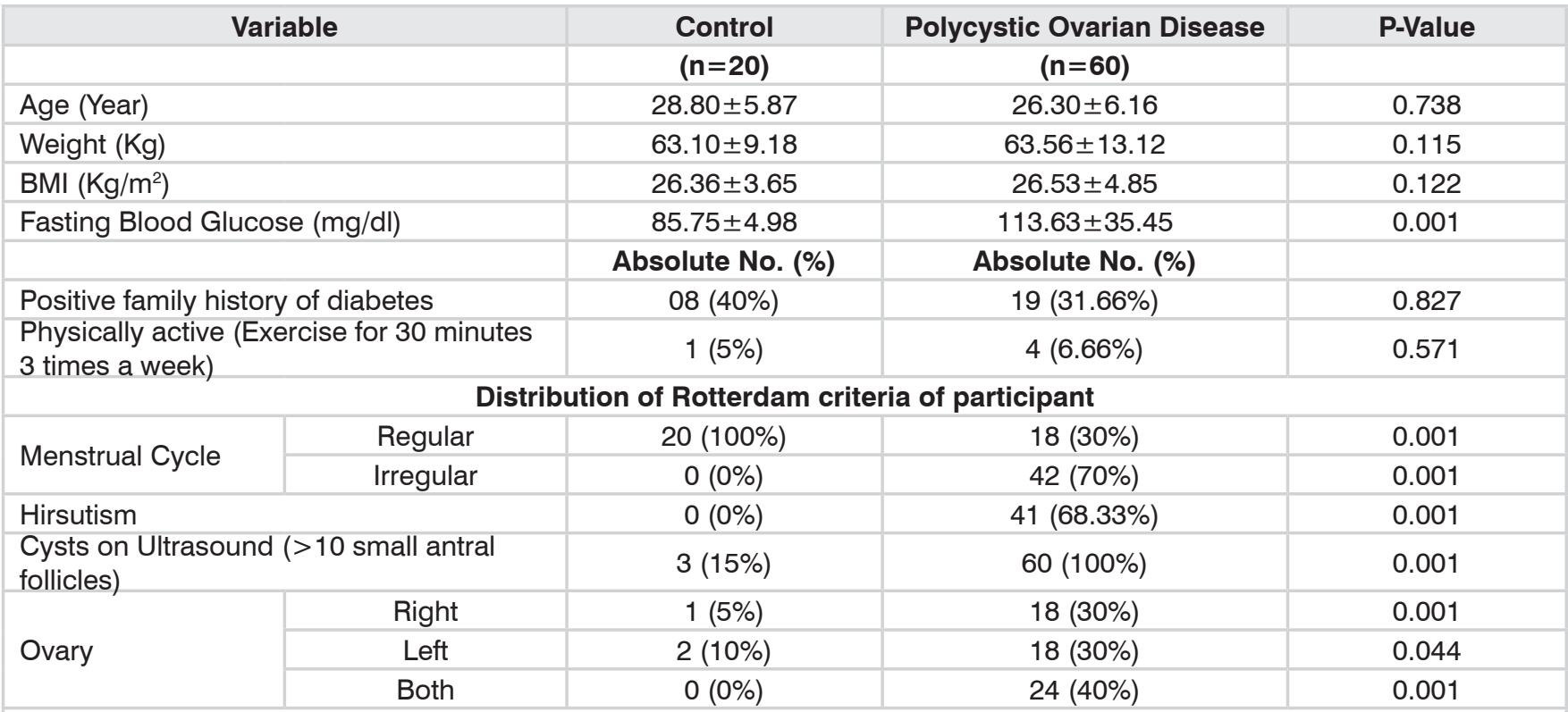

Values expressed as Mean \pm SD and absolute number with percentage in parenthesis. Comparison between groups was made by Student Test and Chi square test. *Statistically significant as compared to controls $p<0.05 .{ }^{* *}$ Statistically significant as compared to compared $p<0.01$

Table-I. Biophysical Data of the Study Cohort.

\begin{tabular}{|l|c|c|c|}
\hline \multicolumn{1}{|c|}{ Variable } & Control & Polycystic Ovarian Disease & P-Value \\
\hline FSH (IU/L) & $\mathbf{( n = 2 0 )}$ & $\mathbf{( n = 6 0 )}$ & 0.114 \\
\hline LH (IU/L) & $6.90 \pm 1.37$ & $6.43 \pm 34.45$ & 0.837 \\
\hline Prolactin ( $\mathrm{ng} / \mathrm{ml})$ & $5.72 \pm 1.41$ & $5.26 \pm 1.12$ & 0.722 \\
\hline Estradiol (pg/ml) & $20.53 \pm 5.83$ & $22.46 \pm 1.28$ & 0.765 \\
\hline Testosterone (pg/ml) & $178.17 \pm 29.74$ & $183.30 \pm 7.04$ & 0.046 \\
\hline Insulin (ulU/ml) & $0.36 \pm 0.19$ & $0.72 \pm 0.27$ & 0.659 \\
\hline Apelin (ng/ml) & $6.59 \pm 11.18$ & $8.58 \pm 1.63$ & 0.000 \\
\hline Cortisol (ng/ml) & $175.20 \pm 23.87$ & $1827.46 \pm 197.28$ & 0.806 \\
\hline
\end{tabular}

Values expressed as Mean \pm SD and absolute number with percentage in parenthesis. Comparison between groups were made by Student Test and Chi square test.

Table-II. Biochemical Data of the study Cohort.

Similar results was reported by another study, where elevated levels of Apelin in PCOS were recorded than healthy women. ${ }^{11}$ Furthermore, when we categorized the study subjects according to BMI; higher apelin levels were seen in subjects with PCO across all BMI categories, suggesting that apelin has a direct effect on ovarian stimulation of follicles. Similar findings was reported by another control study that stated that apelin levels remained the same in all BMI group of PCOS patients. ${ }^{12}$ This study reports higher fasting blood glucose level in PCOS patients than healthy females. This finding is supported by studies conducted by Xianchang et al and OlszaneckaGlinianowicz et al who theorized that in PCOS patient blood glucose level, insulin resistance and BMI are positively affected by level of serum Apelin. ${ }^{13,14}$ Another study supported this idea by showing a positive relationship between the levels of glucose with total cholesterol $(p<0.01)$, triglycerides $(p<0.05)$, LDL-C $(p<0.01)$. There are other studies that reported that blood glucose level, insulin resistance and BMI are positively affected by levels of serum Apelin which may be 
one of the cause of infertility in PCOS females as suggested in our current study. ${ }^{15,16}$

Another important aspect of this study was that $70 \%$ PCO subjects had irregular menstrual cycle and $68 \%$ suffered from hirsutism. Also all PCO subjects had more than 10 antral follicle seen on ultrasound in either one or both ovaries in comparison of $15 \%$ controls $(p<0.05)$. One study reported the presence of apelin receptors in human ovarian cells, follicular fluid and granulosa cells in PCOS subjects than that observed in control patients. ${ }^{17}$ They postulated that apelin is involved in insulin like growth factor 1 (IGF1) induced steroid biosynthesis which ultimately causes increase formation of ovrian cyst in PCOS and confirm APELIN level strong association with ovarian cyst number. Additionally, higher Apelin and Testosterone levels in PCO group was reported when compared with controls $(p<0.05)$, whereas all other markers showed no difference amongst the groups. In another study, PCOS patients showed significantly higher levels of Testosterone compared to healthy controls suggesting a strong link between a high testosterone and adverse metabolic phenotype in PCOS patients. ${ }^{18}$

Besides these promising findings, some of the limitations of this study was its sample size and inability to perform invasive investigation such as ovarian fluid collection to emasure the apelin level. Nevertheless, this study has identified apelin as a possible diagnostic and prognostic biomarker for PCOS patients but further investigations are needed before apelin can be used as treatment for PCOS.

\section{CONCLUSION}

Serum Apelin showed a strong association with irregular menstruation, hirsutism, ovarian cysts, testosterone and deranged blood glucose levels. Therefore, Apelin appears as a potential source for several risk factor in PCOS women of all BMI types.

\section{Acknowledgment}

The authors would like to Ms. Sabah Farhat for her assistance in sample handling and storage.
Copyright@ 26 Oct, 2020.

\section{REFERENCES}

1. Leon LIR, Mayrin JV. Polycystic ovarian disease (Stein-Leventhal Syndrome). Stat Pearls [Internet]: Stat Pearls Publishing; 2018.

2. Bahri Khomami M, Joham AE, Boyle JA, Piltonen T, Arora $\mathrm{C}$, Silagy $\mathrm{M}$, et al. The role of maternal obesity in infant outcomes in polycystic ovary syndrome-A systematic review, meta $\square$ analysis, and meta $\square$ regression. Obesity Reviews. 2019.DOI:10.1111/ obr. 12832.

3. Ding T, Hardiman PJ, Petersen I, Wang F-F, Qu F, Baio $G$. The prevalence of polycystic ovary syndrome in reproductive-aged women of different ethnicity: A systematic review and meta-analysis. Oncotarget. 2017; 8(56):96351.DOI:10.18502/ijrm.v17i8.4818.

4. Witchel SF, Oberfield S, Rosenfield RL, Codner E, Bonny $A$, Ibáñez $L$, et al. The diagnosis of polycystic ovary syndrome during adolescence. Hormone research in paediatrics. 2015; 83(6):376-89. DOI:10.1159/000375530.

5. Condorelli R, Calogero A, Di Mauro M, Cannarella R, Rosta G, La Vignera S. Androgen excess and metabolic disorders in women with PCOS: Beyond the body mass index. Journal of endocrinological investigation. 2018; 41(4):383-8.DOI:10.1007/s40618017-0762-3.

6. Yildiz BO, Yarali $\mathrm{H}$, Oguz $\mathrm{H}$, Bayraktar $\mathrm{M}$. Glucose intolerance, insulin resistance, and hyperandrogenemia in first degree relatives of women with polycystic ovary syndrome. The Journal of Clinical Endocrinology \& Metabolism. 2003; 88(5):2031-6.DOI: 10.1210/jc.2002-021499.

7. Polak K, Czyzyk A, Simoncini T, Meczekalski B. New markers of insulin resistance in polycystic ovary syndrome. Journal of endocrinological investigation. 2017;40(1):1-8.DOI:10.1007/s40618-016-0523-8

8. Dean A, Sullivan K, Soe M. Open source epidemiologic statistics for public health. Version; 2009.

9. Norman RJ, Dewailly D, Legro RS, Hickey TE. Polycystic ovary syndrome. Lancet. 2007; 370(9588):685-97. DOI:10.1016/s0140-6736(07)61345-2.

10. Livadas S, Diamanti-Kandarakis E. Polycystic ovary syndrome: Definitions, phenotypes and diagnostic approach. Front Horm Res. 2013;40:1-21. DOI:10.1159/000341673. 
11. Kiyak Caglayan E, Engin-Ustun Y, Sari N, Gocmen AY, Seckin L, Kara M, et al. Is there association between vitamin D levels, apelin 36, and visfatin in PCOS? Gynecol Endocrinol. 2016; 32(5):386-9. DOI:10.3109/0 9513590.2015.1124260.

12. Ibrahim MKS, Mostafa MI, Abdella RM, Marzouk SA, Elattar S. Relation of serum apelin levels to ultrasound images and Doppler indices in diagnosed polycystic ovary syndrome in overweight and obese women. Middle East Fertility Society Journal. 2018; 23(4):37783.DOI:10.1016/j.mefs.2018.05.007.

13. Sun X, WuX, Zhou Y, YuX, Zhang W. Evaluation of apelin and insulin resistance in patients with PCOS and therapeutic effect of drospirenone-ethinylestradiol plus metformin. Medical science monitor: international medical journal of experimental and clinical research. 2015;21:2547.DOI:10.12659/MSM.894926.

14. Olszanecka $\square$ Glinianowicz M, Madej P, Nylec M, Owczarek A, Szanecki W, Skałba P, et al. Circulating apelin level in relation to nutritional status in polycystic ovary syndrome and its association with metabolic and hormonal disturbances. Clinical Endocrinology. 2013; 79(2):238-42.DOI:10.1111/cen.12120.
15. Choi R, Kim S, Yoo H, Cho Y, Kim S, Chung J, et al. High prevalence of vitamin $D$ deficiency in pregnant Korean women: The first trimester and the winter season as risk factors for vitamin D deficiency. Nutrients. 2015; 7(5):3427-48.10.3390/nu7053427.

16. Yau TT, Ng NY, Cheung LP, Ma RC. Polycystic ovary syndrome: A common reproductive syndrome with long-term metabolic consequences. Hong Kong Med J. 2017; 23(6):622-34.DOI: 10.12809/hkmj176308.

17. Roche J, Rame C, Reverchon M, Mellouk N, Cornuau M, Guerif F, et al. Apelin (APLN) and Apelin receptor (apInr) in human ovary: Expression, signaling, and regulation of steroidogenesis in primary human luteinized granulosa cells. Biol Reprod. 2016; 95(5):104.DOI:10.1095/biolreprod.116.141754.

18. Munzker J, Hofer D, Trummer C, Ulbing M, Harger A, Pieber $\mathrm{T}$, et al. Testosterone to dihydrotestosterone ratio as a new biomarker for an adverse metabolic phenotype in the polycystic ovary syndrome. J Clin Endocrinol Metab. 2015; 100(2):653-60.DOI:10.1210/ jc.2014-2523.

\begin{tabular}{|c|c|c|c|}
\hline \multicolumn{3}{|c}{ AUTHORSHIP AND CONTRIBUTION DECLARATION } \\
\hline Sr. \# & Author(s) Full Name & \multicolumn{1}{|c|}{ Contribution to the paper } & Author(s) Signature \\
\hline 1 & Sassi Kanwal & $\begin{array}{l}\text { Did data collection and manuscript } \\
\text { writing. All authors did review and final } \\
\text { approval of manuscript. } \\
\text { Did data collection and manuscript } \\
\text { writing. All authors did review and final } \\
\text { approval of manuscript. } \\
\text { Manuscript writing, All authors } \\
\text { did review and final approval of } \\
\text { manuscript. } \\
\text { Conceived, designed and did statistical } \\
\text { analysis \& editing of manuscript. All } \\
\text { authors did review and final approval } \\
\text { of manuscript. }\end{array}$ \\
\hline 3 & Ashba Allahwasaya & Sargis Anjum & Syeda Sadia Fatima
\end{tabular}

